\title{
A DNA vaccine encoding mutated HPV58 mE6E7-Fc-GPI fusion antigen and GM-CSF and B7.I
}

This article was published in the following Dove Press journal:

OncoTargets and Therapy

23 October 2015

Number of times this article has been viewed

\section{He Wang' \\ Jiyun $\mathrm{Yu}^{2}$ \\ $\mathrm{Li} \mathrm{Li'}$}

'Department of Gynecologic Oncology, Affiliated Tumor Hospital of Guangxi Medical University, Nanning, Guangxi, ${ }^{2}$ Institute of Basic Medical Sciences, Academy of Military Medical Sciences, Beijing, People's Republic of China
Correspondence: Li Li

Department of Gynecologic Oncology,

Affiliated Tumor Hospital of Guangxi

Medical University, 7I He Di Road,

Nanning 53002I, Guangxi, People's

Republic of China

Tel +867715310430

Email lili_temp@hotmail.com
Background: Persistent infection with high-risk human papillomavirus (HPV) is a predominant cause of cervical cancer, and HPV58 is the third most common virus detected in the patients with cervical cancer in Asia. E6 and E7 are the viral oncogenes which are constitutively expressed in HPV-associated tumor cells and can be used as target antigens for related immunotherapy. In this study, we modified the HPV58 E6 and E7 oncogenes to eliminate their oncogenic potential and constructed a recombinant DNA vaccine that coexpresses the sig-HPV58 mE6E7-Fc-GPI fusion antigen in addition to granulocyte-macrophage colony-stimulating factor (GM-CSF) and B7.1 as molecular adjuvants (PVAX1-HPV58 mE6E7FcGB) for the treatment of HPV58 (+) cancer. Methods: PVAX1-HPV58 mE6E7FcGB recombinant DNA vaccine was constructed to express a fusion protein containing a signal peptide, a modified HPV58 mE6E7 gene, and human IgG Fc and glycosylphosphatidylinositol (GPI)-anchoring sequences using the modified DNA vaccine vector PVAX1-IRES-GM/B7.1 that coexpresses GM-CSF, and B7.1. C57BL/6 mice were challenged by HPV58 E6E7-expressing B16-HPV58 E6E7 cells, followed by immunization by PVAX1-HPV58 mE6E7FcGB vaccine on days 7, 14, 21 after tumor challenge. The cellular immune responses in immunized mice were assessed by measuring IFN- $\gamma$ production in splenocytes upon stimulation by HPV58 E6E7-GST protein and the lysis of B16-HPV58 E6E7 target cells by splenocytes after restimulation with HPV58 E6E7-GST protein. The antitumor efficacy was evaluated by monitoring the growth of the tumor.

Results: PVAX1-HPV58 mE6E7FcGB elicited varying levels of IFN-lsgdB58onn T-cell immune responses and lysis of target cell in mice in response to the recombinant antigen HPV58 E6E7-GST. Furthermore, the vaccine also induced antitumor responses in the HPV58 (+) B16HPV58 E6E7 tumor challenge model as evidenced by delayed tumor development.

Conclusion: The recombinant DNA vaccine PVAX1-HPV58 mE6E7FcGB efficiently generates cellular immunity and antitumor efficacy in immunized mice. These data provide a basis for the further study of this recombinant vaccine as a potential candidate vaccine.

Keywords: human papillomavirus type 58, E6 gene, E7 gene, DNA vaccine, immunogenicity

\section{Introduction}

Cervical cancer is the second most common cancer in women worldwide and the most common cancer in women in most developing countries. ${ }^{1}$ Strong molecular epidemiological evidence indicates that persistent infection with high-risk human papillomavirus (HPV) is the major cause of invasive cervical cancer and cervical intraepithelial neoplasia, and HPV can be detected in $99.7 \%$ of cervical cancer patients. ${ }^{2}$ Two prophylactic HPV vaccines, Gardasil, a quadrivalent HPV16/18/6/11 virus-like particles vaccine ${ }^{3}$ from Merck \& Co., Inc. (NJ, USA), and another bivalent HPV16/18 virus-like particles vaccine from GlaxoSmithKline plc (London, UK), 
Cervarix, ${ }^{4}$ have been confirmed to prevent most high-risk HPV infections and to minimize the consequences of HPVassociated diseases. However, these vaccines have been shown to be effective only in the girls from 11 to 12 years, according to FDA recommendation, without previous HPV infection history and have not shown a therapeutic effect against current HPV infection or associated lesions. ${ }^{5} \mathrm{~A}$ large population will remain at risk of HPV infection in the years to come. Therefore, the development of a therapeutic vaccine targeting high-risk HPV is important. Most recent studies have focused on therapeutic vaccines against HPV16, which causes approximately $46 \%-63 \%$ of squamous cell carcinomas worldwide, ${ }^{6}$ therefore, the immunodominant epitopes E6 and E7 for HPV16 and their associated immune responses have been well characterized. ${ }^{7-9}$ For HPV 58, which is the third most common virus in cervical cancer cases in Asia, ${ }^{10}$ limited data are available regarding therapeutic vaccines. Therefore, developing a therapeutic vaccine targeting HPV58 is necessary for these countries.

In the open reading frames encoding proteins of HPV, early proteins (E) were involved in cellular transformation, and DNA transcription and replication. E6 and E7 are the HPV oncoproteins and are constitutively expressed in HPVassociated cells, and their expression is necessary for the transformation and maintenance of the malignant phenotype of the cells. ${ }^{11,12}$ Therefore, E6 and E7 are usually used as target antigens for HPV therapeutic vaccines. However, potential transgene integration to the host genome may increase carcinogenic risk of the host cells. To reduce this risk, here, we inactivated the confirmed coding regions of HPV58 E6 and E7 responsible for oncogenic transformation. ${ }^{13,14}$

In this study, we modified the HPV58 E6 and E7 oncogenes to eliminate their oncogenic potential and constructed a recombinant DNA vaccine that coexpresses the sig-HPV58 mE6E7-Fc- GPI fusion antigen and granulocyte-macrophage colony-stimulating factor (GM-CSF) ${ }^{15,16}$ and B7.1 as molecular adjuvants ${ }^{17,18}$ (PVAX1-HPV58 mE6E7FcGB) for use as a therapeutic vaccine for the treatment of HPV58 (+) cancer.

\section{Materials and methods Cell lines}

Human embryonic kidney 293T cells, mouse melanoma B16 cells, and mouse embryonic fibroblast NIH/3T3 cells were purchased from the Cell Bank of the Chinese Academy of Sciences (Beijing, People's Republic of China). The cells were cultured in RPMI (Roswell Park Memorial Institute)-1640 medium and Dulbecco's Modified Eagle's Medium (HyClone; Logan, UT, USA) supplemented with 10\% fetal bovine serum (HyClone), $2 \mathrm{mM}$ glutamine, $1 \mathrm{mM}$ sodium bicarbonate, and $100 \mathrm{U} / \mathrm{mL}$ penicillin and $100 \mu \mathrm{g} / \mathrm{mL}$ streptomycin. The cells were maintained in a humidified air chamber containing $5 \% \mathrm{CO}_{2}$ at $37^{\circ} \mathrm{C}$.

\section{Mice}

Fifty 6-to-8 week-old female C57BL/6 mice were purchased from the Animal Breeding Center of Military Medical Science (Beijing, People's Republic of China). All mice were raised under specific pathogen-free conditions, and all procedures were performed according to general protocols for the proper use and care of laboratory animals. All mice experiments were approved by the ethics committee of Affiliated Tumor Hospital of Guangxi Medical University.

\section{Mutagenesis and fusion of HPV58 E6 and E7}

HPV58 E6 and E7 genes were modified to inactivate their transforming activity and then fused into one reading frame. According to the literature, the amino acids mutations at $50 \mathrm{~L}$, $63 \mathrm{C}$, and $106 \mathrm{C}$ in $\mathrm{E} 6$ have been shown to remove the ability to degrade p53 and inactivate its oncogenic activity, ${ }^{19,20}$ and the E7 amino acids at positions 24C, 26E, and 92C were mutated to abolish retinoblastoma $(\mathrm{Rb})$ binding and degradation. ${ }^{20-22}$ For all mutations, the wild-type amino acids were mutated to glycine. In addition, for vaccine plasmid transcription, the E6 and E7 mutations were deleted their termination signal and fused into one reading frame without altering their amino acid sequences. The modified HPV58 E6E7 fusion gene was named mE6E7. The HPV58 mE6E7 fusion fragment was commercially synthesized (Qingke Biologic Technology Company, Beijing, People's Republic of China) and cloned into the pGEM-T easy vector (Takara, Dalian, People's Republic of China) to generate the pGEM-T easy-HPV58 mE6E7 plasmid. The presence of the HPV58 mE6E7 gene in the plasmid was verified by sequencing analysis.

\section{Construction of the PVAXI-HPV58 $\mathrm{mE6}$ E7FcGB vaccine plasmid and immunofluorescence staining}

To construct the vaccine plasmid, HPV58 mE6E7 was excised from the plasmid pGEM-T easy-HPV58 mE6E7 by digestion with Xho I and EcoR I (New England Biolabs, Ipswich, MA, USA) and cloned into the PCI-sig-Fc-GPI vector, giving rise to PCI-sig-HPV58 mE6E7-Fc-GPI. Subsequently, the sig-HPV58 mE6E7-Fc-GPI fused expression cassette was excised from the PCI-sig-HPV58 mE6E7-Fc-GPI plasmid by double digestion with Nhe I and Not I and was inserted upstream of the internal ribosome entry site (IRES) of the PVAX1-IRES-GM/B7.1 vector at the Nhe I and Cla I sites to create PVAX1-HPV58 mE6E7FcGB. 
One day prior to transfection, 293T cells were seeded at a density of $4 \times 10^{5}$ cells per well in 6-well plates in $2 \mathrm{~mL}$ of Opti-MEM medium (Thermo Fisher Scientific, Waltham, MA, USA). The cells were transfected with $4 \mu \mathrm{g}$ of PVAX1-HPV58 $\mathrm{mE} 6 \mathrm{E} 7 \mathrm{FcGB}$ plasmid DNA using $10 \mu \mathrm{L}$ of Lipofectamine ${ }^{\mathrm{TM}}$ 2000 (Invitrogen) reagent according to the manufacturer's instructions (Invitrogen). The transfected cells were collected after 48 hours and washed three times with phosphate-buffered saline (PBS), and then concurrently incubated with a rabbit anti-human IgG-FITC (immunoglobulin G-conjugated fluorescein isothiocyanate) antibody (1:100, 11-0168, eBioscience, San Diego, CA, USA) and a mouse anti-human B7-1phycoerythrin (PE) antibody (1:100, 12-0809, eBioscience) at $4^{\circ} \mathrm{C}$ for 2 hours. After extensive washing with PBS, the cells were scanned using a laser-scanning confocal microscope.

\section{Expression and purification of the HPV58 E6E7-GST fusion protein}

The HPV58 E6E7 fusion fragment was amplified by polymerase chain reaction. The $\mathrm{pET}-42 \mathrm{a}(+)$ prokaryotic expression vector and the HPV58 E6E7 fragment were both cut with EcoR I and Xho I and then linked together to produce the recombinant plasmid pET-42a(+)-HPV58 E6E7. The recombinant plasmid was amplified and expressed in Escherichia coli BL21 cells. The HPV58 E6E7-GST fusion protein was expressed by induction with isopropyl $\beta$-D-1-thiogalactopyranoside (IPTG, Promega Corp., Madison, WI, USA) and purified using glutathione agarose resin (Promega Corp.).

\section{Establishment of an HPV58 E6E7- expressing B16-HPV58 E6E7 melanoma cell line}

To establish an HPV58 E6E7-expressing cell line, mouse melanoma B16 cells were transfected with pIRES-neo/ HPV58 E6E7 using the Lipofectamine ${ }^{\mathrm{TM}} 2000$ (Invitrogen). G418-resistant cell clones stably expressing the HPV58 E6E7 fusion protein were selected using a medium containing G418 at a concentration of $1,000 \mu \mathrm{g} / \mathrm{mL}$. Then, the HPV58 E6E7-expressing B16-HPV58 E6E7 cell line was identified by immunofluorescence and Western blot analysis.

\section{Immunization of mice}

Fifty female C57BL/6 mice, 6-8 weeks old, were randomly divided into five groups as follows: sham treatment group (PBS), PVAX1 group, PVAX1-IRES-GM/B7.1 group, PVAX1-HPV58 mE6E7Fc group, and PVAX1-HPV58 $\mathrm{mE6E7FcGB}$ vaccine group. Sham-treated mice were intramuscularly injected with $100 \mu \mathrm{L}$ of PBS. The remaining four groups of mice were immunized by intramuscular injection of $50 \mu \mathrm{g}$ of purified PVAX1, PVAX1-IRES-GM/B7.1, PVAX1HPV58 mE6E7Fc, or PVAX1-HPV58 mE6E7FcGB plasmids. All of the mice were intramuscularly immunized with the same dose three times at 7-day intervals (Figure 1).

\section{Tumor challenge and in vivo tumor growth}

C57BL/6 mice were subcutaneously injected with $1 \times 10^{5}$ HPV58 E6E7-expressing B16-HPV58 E6E7 cells. The PVAX1-HPV58 mE6E7FcGB DNA vaccine was subsequently administered by tattooing on days 7,14 , and 21 after tumor challenge. Tumor growth was monitored twice each week using caliper measurements in two dimensions. The volume of the tumors was calculated as follows:

$$
\text { volume }=\left(\text { width }^{2} \times \text { length }\right) / 2
$$

The mice were sacrificed when the tumor diameter reached $15 \mathrm{~mm}$ or when the tumor volume exceeded $1,000 \mathrm{~mm}^{3} .{ }^{33}$

\section{Measurement of IFN- $\gamma$ production using the ELISPOT assay}

Two weeks after the booster inoculations, HPV58 E6E7specific interferon (IFN)- $\gamma$ production by splenocytes isolated from individual mice following immunization was detected using an ELISPOT kit (BD Bioscience, San Diego, CA, USA) according to the instructions given in the manufacturer's manual with minor modifications. Briefly, a 96-well nitrocellulose plate was coated with 10 $\mu \mathrm{g} / \mathrm{mL}$ rat anti-mouse IFN- $\gamma$ antibody in $100 \mu \mathrm{L}$ of PBS and incubated overnight at $4^{\circ} \mathrm{C}$. The plates were washed and blocked with RPMI-1640 medium supplemented with

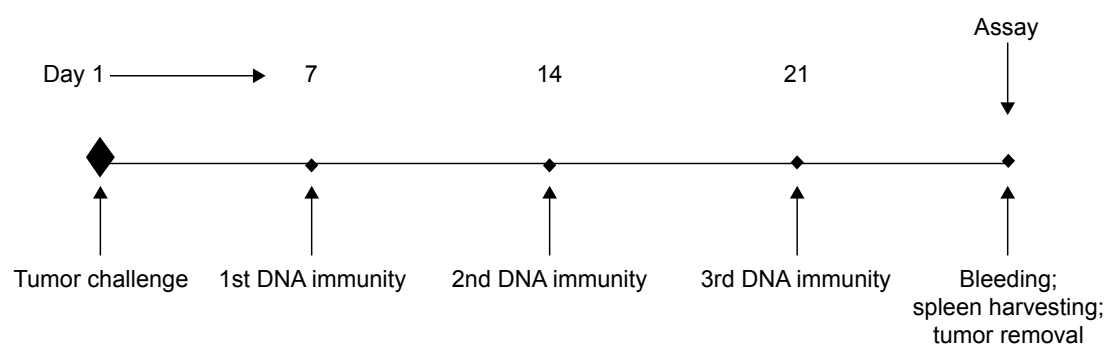

Figure I Illustration of timeline of experiments. 
$10 \%$ fetal bovine serum. Splenocytes were added to the plate at an initial concentration of $4 \times 10^{5}$ cells/well in a volume of $100 \mu \mathrm{L}$. The cells were stimulated with HPV58 E6E7-GST protein $(10 \mu \mathrm{g} / \mathrm{mL})$ or an irrelevant peptide (10 $\mu \mathrm{g} / \mathrm{mL}$, irrelevant peptide was diluted with PBS 50 $\mathrm{mL}$ and added to the splenocytes). Phorbol-12-myristate 13-acetate (PMA, $5 \mathrm{ng} / \mathrm{mL}$ ) served as a positive control, and an irrelevant peptide and media alone served as negative controls. The plate was incubated overnight at $37^{\circ} \mathrm{C}$ in $5 \% \mathrm{CO}_{2}$. The next day, the cells were removed by washing the plate twice with deionized $\mathrm{H}_{2} \mathrm{O}$ and thrice with PBS- $0.05 \%$ Tween 20, with each wash performed for 5 minutes. Then, $4 \mu \mathrm{g} / \mathrm{mL}$ of biotinylated rat anti-mouse IFN- $\gamma$ antibody in $100 \mu \mathrm{L}$ of PBS was added to each well, and the plate was incubated for 2 hours at $37^{\circ} \mathrm{C}$. The wells were washed three times with PBS-0.05\% Tween 20 for 2 minutes, and then incubated for 1 hour with $100 \mu \mathrm{L}$ of a 1:100 dilution of the enzyme conjugate (streptavidin-HRP) in $\mathrm{PBS}$ at $37^{\circ} \mathrm{C}$ in $5 \% \mathrm{CO}_{2}$. After the plate had been washed four times with PBS- $0.05 \%$ Tween 20 for 2 minutes, followed by two washes with PBS alone, spots were developed by adding $100 \mu \mathrm{L}$ of 3-amino-9-ethylcarbazole (AEC) and incubating the plate at room temperature for 30 minutes. The plate was washed and allowed to air dry overnight. The spots, representing individual IFN- $\gamma$-producing cells as spot-forming cells (SFCs), on the membrane were counted using an Eli. Scan ELISPOT scanner (A.EL.VIS, Hannover, Germany; software version 4.1).

\section{Cytotoxic T-lymphocyte (CTL) assay}

Splenocytes isolated from immunized mice were cultured with HPV58 E6E7-GST protein and interleukin-2 (IL-2, $20 \mathrm{U} / \mathrm{mL}$ ) for 5 days to serve as effector cells. B16-HPV58 E6E7 cells, used as target cells $\left(1 \times 10^{4}\right.$ per well), were incubated with washed effector cells at various effector-to-target cell (E/T) ratios (10:1, 20:1, and 40:1). Nontransfected B16 cells served as a negative control. After 6 hours of coculture at $37^{\circ} \mathrm{C}, 50 \mu \mathrm{L}$ of the centrifuged supernatant was collected to quantitatively assess the amount of lactate dehydrogenase (LDH) using CytoTox 96 nonradioactive cytotoxicity assay kits (Promega Corp.) according to the manufacturer's instrtuctions. The lytic activity of the T-cells was calculated according to the following formula using the mean value from triplicate wells. ${ }^{24}$
Table I Gene changes used to inactivate the oncogene function of HPV58 E6

\begin{tabular}{lll}
\hline aa & HPV58 E6 & HPV58 mE6 \\
\hline 50 & TTA(L) & GGT(G) \\
63 & TGT(C) & GGT(G) \\
106 & TGT(C) & GGT(G) \\
\hline
\end{tabular}

Abbreviations: aa, amino acid; HPV, human papillomavirus.

\section{Statistical analysis}

Statistical analysis of animal survival was performed using SPSS for Windows (version 13.0; SPSS Inc., Chicago, IL, USA). Results are expressed as mean \pm standard deviation. Data from the IFN-ELISPOT assay and tumor treatment experiments were evaluated using analysis of variance. To identify significant differences between groups, Student's $t$-test was used. For all comparisons, differences were considered significant when $P<0.05$. All findings were confirmed in at least one additional independent experiment.

\section{Results \\ E6E7 mutations and the loss of oncogenic activity in $\mathrm{mE6E7}$}

To reduce the risk associated with the HPV58 E6 and E7 proteins, six known oncogenic regions within E6 and E7 were mutated (Tables 1 and 2, Figure 2A). In addition, E6 and E7 were fused without altering the amino acid sequence. To confirm the expression of the HPV58 E6E7 and HPV58 mE6E7 fusion proteins, NIH/3T3 cells were transfected with the plasmids pIRES-neo/ HPV58 E6E7, pIRES-neo/HPV58 mE6E7, and pIRES-neo. The positive cell clones were subsequently selected with medium containing G418 at $400 \mu \mathrm{g} / \mathrm{mL}$. Western blot analysis of the NIH/3T3-HPV58 E6E7, NIH/3T3-HPV58 mE6E7, and NIH/3T3-neo cells confirmed HPV58 E6E7 and HPV58 mE6E7 protein expression (Figure 2B). A transformation assay in soft agar showed that NIH/3T3-HPV58 E6E7 cells grew in colonies, whereas NIH/3T3-neo and NIH/3T3-HPV58 mE6E7 cells grew in a nonadherent manner. These data suggest that, in contrast to HPV58 E6E7, HPV58 mE6E7 induces morphological changes related to the loss of cellular transformation (Figure 2C).

\section{Construction and expression of the recombinant PVAXI-HPV58 mE6E7FcGB DNA vaccine}

The construction of the recombinant PVAX1-HPV58 $\mathrm{mE6E7FcGB} \mathrm{DNA} \mathrm{vaccine} \mathrm{plasmid} \mathrm{is} \mathrm{presented} \mathrm{in} \mathrm{Figure} \mathrm{3A.}$

Percent cytotoxicity $=\frac{\text { Experimental value }- \text { Effector spontaneous value }- \text { Target spontaneous value }}{\text { Target maximum value }- \text { Target spontaneous value }} \times 100 \%$ 
Table 2 Gene changes used to inactivate the oncogene function of HPV58 E7

\begin{tabular}{lll}
\hline aa & HPV58 E7 & HPV58 $\mathbf{m E 7}$ \\
\hline 24 & TGC(C) & GGC(G) \\
26 & GAG(E) & GGG(G) \\
92 & TGC(C) & GGC(G) \\
\hline
\end{tabular}

Abbreviations: aa, amino acid; HPV, human papillomavirus.

PVAX1-HPV58 mE6E7FcGB plasmid DNA was digested with Nhe I and Pvu I. The plasmid was subsequently verified by sequencing. After 48 hours of transient transfection with PVAX1-HPV58 mE6E7FcGB plasmid 293T cells, green and red fluorescence were seen on the cell membrane indicative of simultaneous expression of the sig-HPV58 mE6E7-Fc-GPI (green) and GM-CSF/B7.1 (red) fusion proteins (Figure 3B).

\section{Establishment of the HPV58 E6E7- expressing B16-HPV58 E6E7 cell line}

The pIRES-neo/HPV58 E6E7 plasmid was transfected into B16 cell line. After selection with medium containing G418, the positive cell clones showed green fluorescence localized to the cytoplasm (Figure 4A). The HPV58 E6E7 protein was also detected by Western blotting (Figure 4B). These results indicate that the B16-HPV58 E6E7 cell line could be used as an HPV58 E6E7-expressing cell line.

\section{Expression and purification of the HPV58 E6E7-GST fusion protein}

The HPV58 E6E7-GST fusion protein was successfully expressed in E. coli BL21 cells after transformation with the recombinant pET-42a (+)-HPV58 E6E7 plasmid. Because the expressed recombinant protein contained a GST tag, it could be purified using glutathione agarose resin. The size of the HPV58 E6E7-GST fusion protein product, predicted to be $55 \mathrm{kDa}$, was confirmed by Coomassie Blue staining and Western blot analysis (Figure 5).

\section{Antitumor efficacy of PVAXI-HPV58 $m E 6 E 7 F c G B$ in the Bl6-HPV58 E6E7 tumor mouse model}

To investigate whether treatment with the PVAX1-HPV58 $\mathrm{mE6E7FcGB}$ vaccine has antitumor effects in vivo, the growth of B16-HPV58 E6E7 tumor cells was monitored every 2 days after challenge. The tumor growing curve of mice immunized with PVAX1-HPV58 mE6E7FcGB was the lowest among the five groups of mice (Figure 6A). We measured the mean ( \pm standard deviation) weight of the tumors in mice challenged with HPV58 E6E7-expressing B16-HPV58 E6E7 cells. Our data indicate that the mean weight of the tumors was $1.40 \pm 0.52 \mathrm{~g}$ for the PBS group, $1.50 \pm 0.60 \mathrm{~g}$ for the PVAX1 group, $1.62 \pm 0.31 \mathrm{~g}$ for the PVAX1-IRES-GM/B7.1 group,
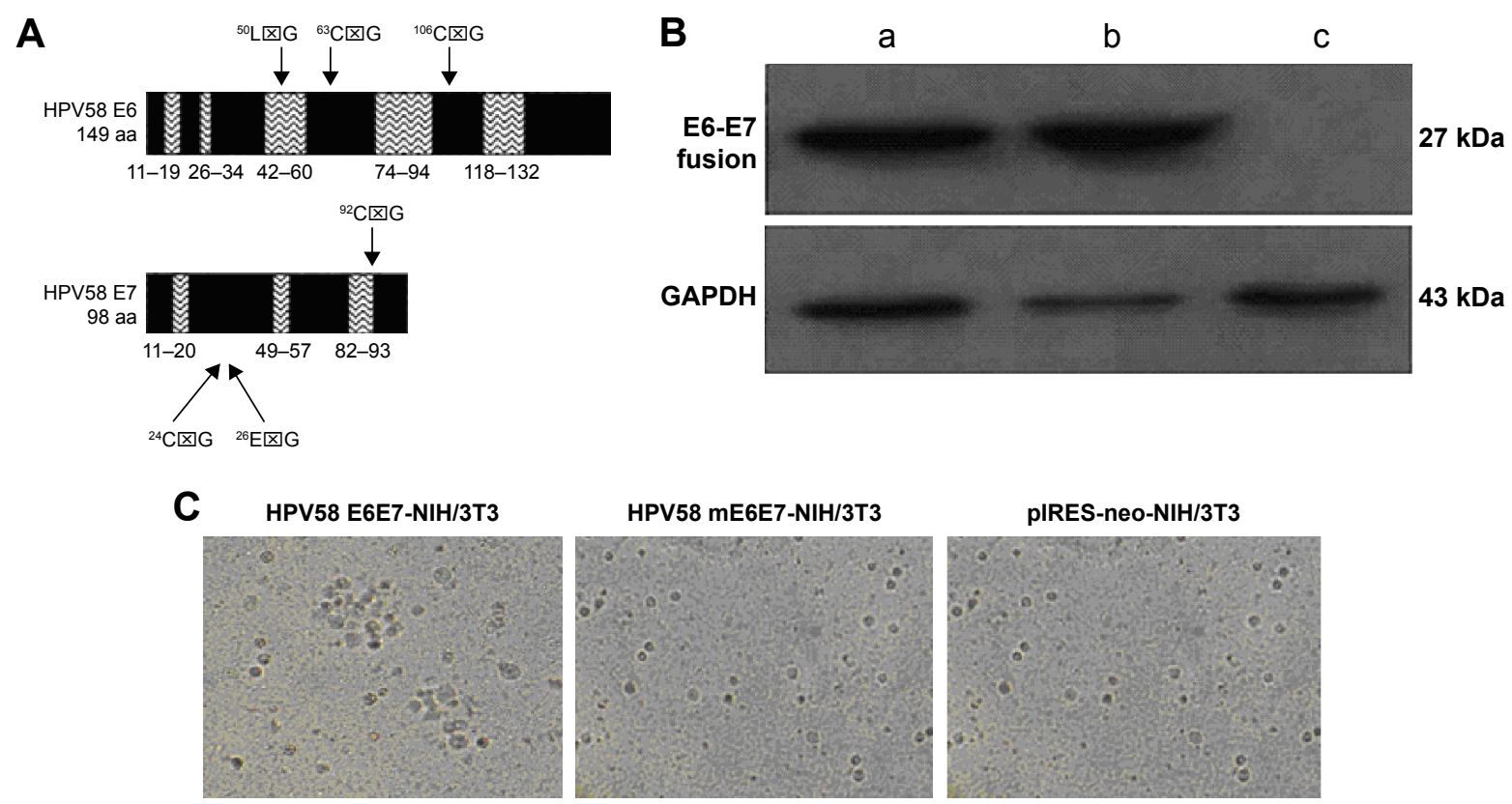

Figure 2 Gene changes used to inactivate the oncogene function of HPV58 E6E7.

Notes: (A) Locations of the point mutations introduced into the HPV58 E6 and E7 proteins. The naturally occurring amino acids E6-50L, 63C, and I06C and E7-24C, 26E, and 92C were mutated to glycine. (B) Western blot analysis showed that HPV58 E6E7 and HPV58 mE6E7 fusion proteins can be expressed in NIH/3T3-HPV58 E6E7 (a) and NIH/3T3-HPV58 mE6E7 (b) cells, but not in NIH/3T3-neo cells (c). GAPDH served as a protein loading control. (C) Colony formation in soft agar about three stable transfected $\mathrm{NIH} / 3 \mathrm{~T} 3$ cell lines.

Abbreviations: HPV, human papillomavirus; GAPDH, glyceraldehyde 3-phosphate dehydrogenase; $囚$, TTA(L)- Leucine, TGT(C)-Cysteine, GGT(G)-Glycine, TGC(C)Cysteine, GAG(E)-Glutamic acid, GGC(G)-Glycine, GGG(G)-Glycine. 


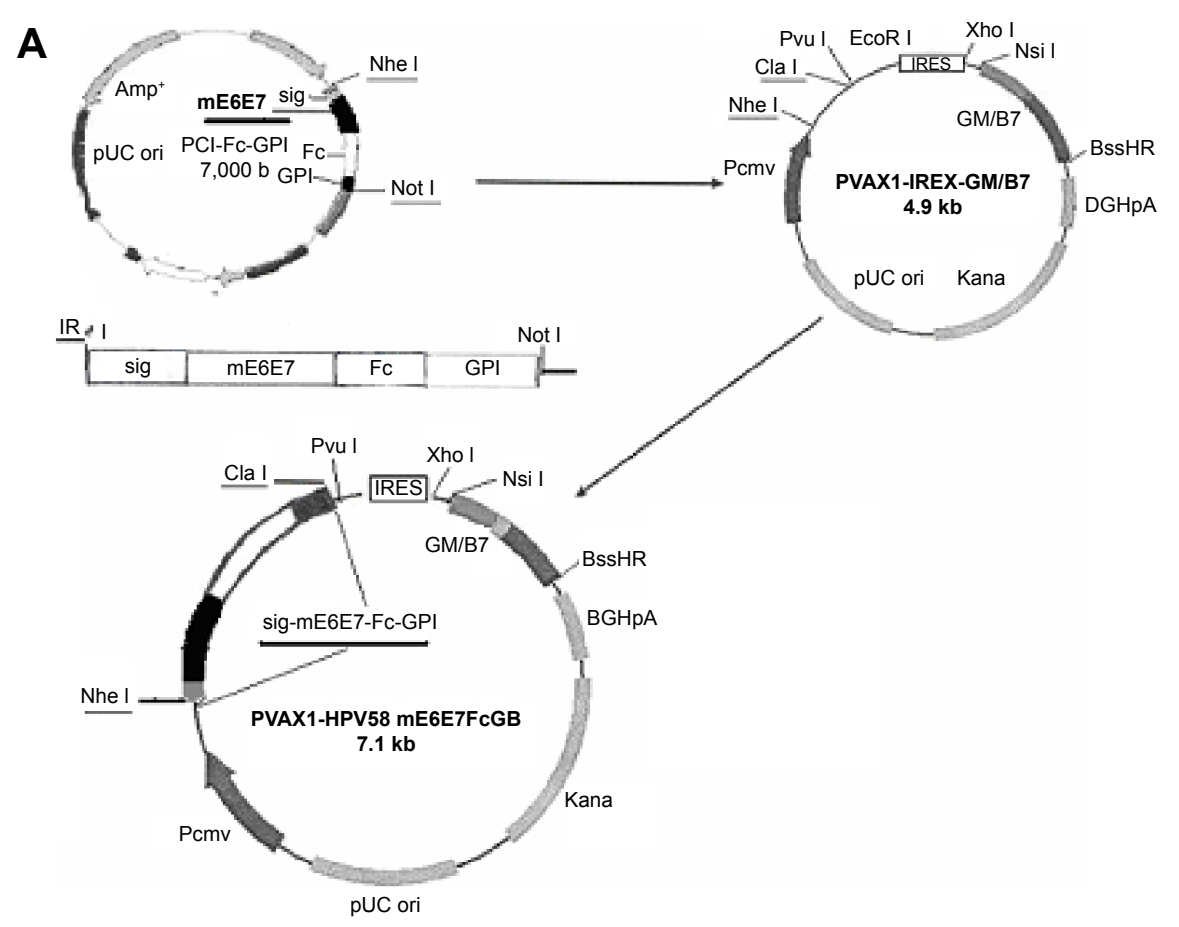

B a
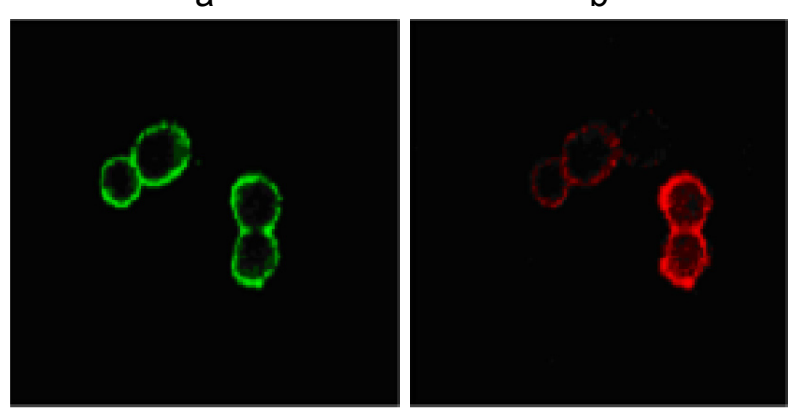

Figure 3 Construction of the PVAXI-HPV58 mE6E7FcGB plasmid and expression of the target proteins assessed by immunofluorescence staining. Notes: (A) Diagram of the construction of the recombinant DNA vaccine PVAXI-HPV58 mE6E7FcGB. HPV58 mE6E7 was cloned into the plasmid PCl-sig-Fc-GPI, and the fused cassette sig-HPV58 mE6E7-Fc-GPI was subsequently excised from PCl-sig-HPV58 mE6E7-Fc-GPI plasmid and inserted into the upstream of IRES in PVAXI-IRES-GM/B7.I vector to create PVAXI-HPV58 mE6E7FcGB. (B) The transfected 293T cells were concurrently incubated with a rabbit anti-human IgG-FITC antibody (green) and a mouse anti-human B7-I-PE antibody (red). Immunofluorescence revealed the expression of sig-HPV58 mE6E7-Fc-GPI (a, green, $\times 50)$ and GM-CSF/B7.I (b, red, $\times 50)$ at the cells membrane. Abbreviations: IgG-FITC, immunoglobulin G-conjugated fluorescein isothiocyanate; IRES, internal ribosome entry site; HPV, human papillomavirus.

A

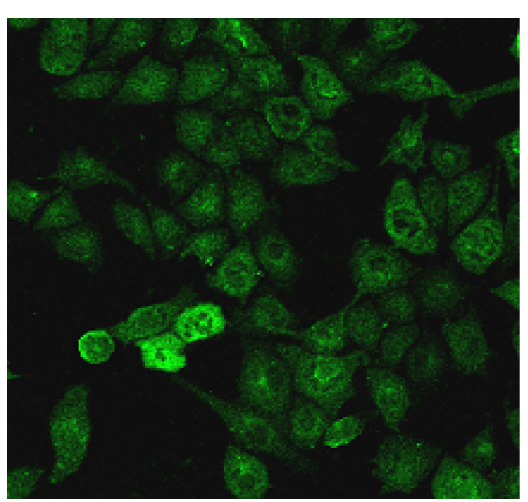

B

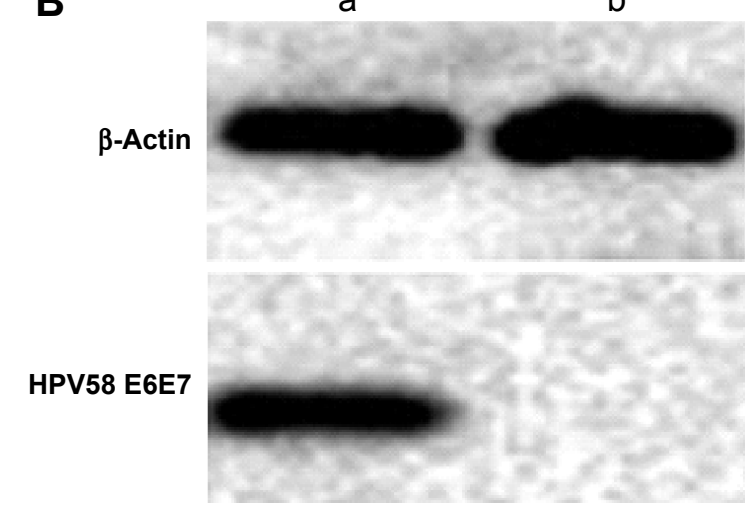

Figure 4 The pIRES-neo/HPV58 E6E7 plasmid was transfected into BI6 cell line.

Notes: (A) Immunofluorescence assays in stable transfected pIRES-neo-HPV58 E6E7 BI6 cell line. (B) Western blot results of transient expression of HPV58 E6E7 in BI6 cell line. (a) The experimental group transfected pIRES-neo-HPV58 E6E7 and (b) the control group. 


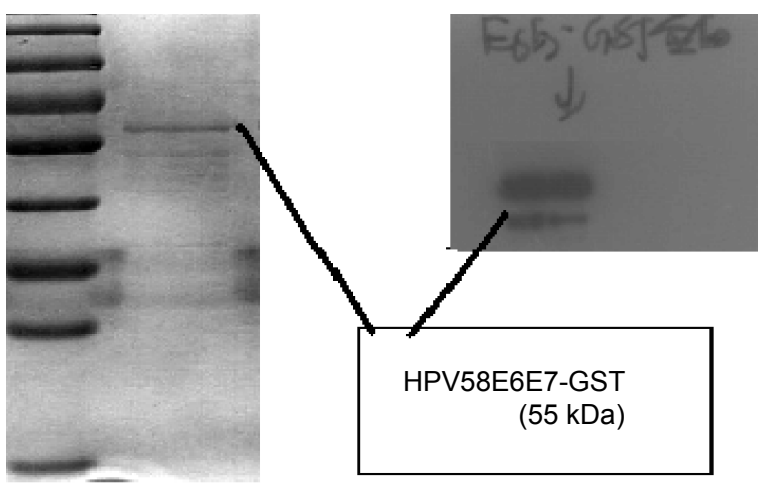

Figure 5 SDS-PAGE and western blot analysis of purified HPV58 E6E7 fusion protein $(55 \mathrm{kDa})$.

Abbreviations: SDS-PAGE, sodium dodecyl sulfate-polyacrylamide gel electrophoresis; HPV, human papillomavirus.

and $0.66 \pm 0.34 \mathrm{~g}$ for the PVAX1-HPV58 mE6E7Fc group. The tumors in all of these groups were larger than those in the PVAX1-HPV58 mE6E7FcGB vaccine group $(0.26 \pm 0.11 \mathrm{~g})$, and these differences were significant $(P<0.01)$ (Figure 6B).

\section{T-cell immune responses against the HPV58 E6E7-GST protein after immunization with PVAXI-HPV58 mE6E7FcGB in mice}

To test HPV58-specific T-cell immune responses induced by the recombinant PVAX1-HPV58 mE6E7FcGB vaccine, C57BL/6 mice were injected with PVAX1-HPV58 mE6E7FcGB, PVAX1-HPV58 mE6E7Fc, PVAX1IRES-GM/B7.1, PVAX1, or PBS. The splenocytes from immunized mice were tested directly ex vivo in IFN- $\gamma$ Elispot assays against the HPV58 E6E7-GST protein. PVAX1-HPV58 mE6E7FcGB induced specific cellular immune responses against the HPV58 E6E7-GST protein in $\mathrm{C} 57 \mathrm{BL} / 6$ mice after three immunizations, and the average number of IFN- $\gamma$-positive spots was $218.75 \pm 34.42$ $\mathrm{SFC} / 4 \times 10^{5}$ splenocytes. The positive spots were significantly increased compared with those of the control mice immunized with PVAX1-HPV58 mE6E7Fc (138.61 \pm 17.54 SFC/4 $\times 10^{5}$ splenocytes, $\left.P<0.05\right)$, PVAX1-IRES-GM/B7.1 (27.61 $\pm 5.37 \mathrm{SFC} / 4 \times 10^{5}$ splenocytes, $P<0.01$ ), PVAX1 (13.33 $\pm 1.53 \mathrm{SFC} / 4 \times 10^{5}$ splenocytes, $\left.P<0.001\right)$, or PBS (5.62 $\pm 4.78 \mathrm{SFC} / 4 \times 10^{5}$ splenocytes, $P<0.001$ ) (Figure 7).

\section{CTLs induced by vaccination with PVAXI-HPV58 mE6E7FcGB}

To perform a CTL assay, splenocytes were isolated from vaccinated mice. After restimulation with the HPV58 E6E7-GST protein, the splenocytes served as effector cells, and HPV58 E6E7-expressing B16-HPV58 E6E7 cells served as target cells. ${ }^{8}$ As shown in Figure 8, splenocytes from mice vaccinated with PVAX1-HPV58 mE6E7FcGB $(63.4 \% \pm 3.3 \%)$ had significantly higher specific cytolytic activity at an $\mathrm{E} / \mathrm{T}$ ratio of 40:1 than mice vaccinated with PVAX1-HPV58 mE6E7Fc (31.1\% $\pm 2.75 \%)$, PVAX1-IRES-GM/B7.1 (22.1\% $\pm 0.99 \%)$, PVAX1 $(9.4 \% \pm 0.39 \%)$, or PBS $(5.3 \% \pm 2.2 \%)(P<0.05)$. The differences between all of these groups at $\mathrm{E} / \mathrm{T}$ ratios of 20:1 or 10:1 did not reach overall statistical significance.
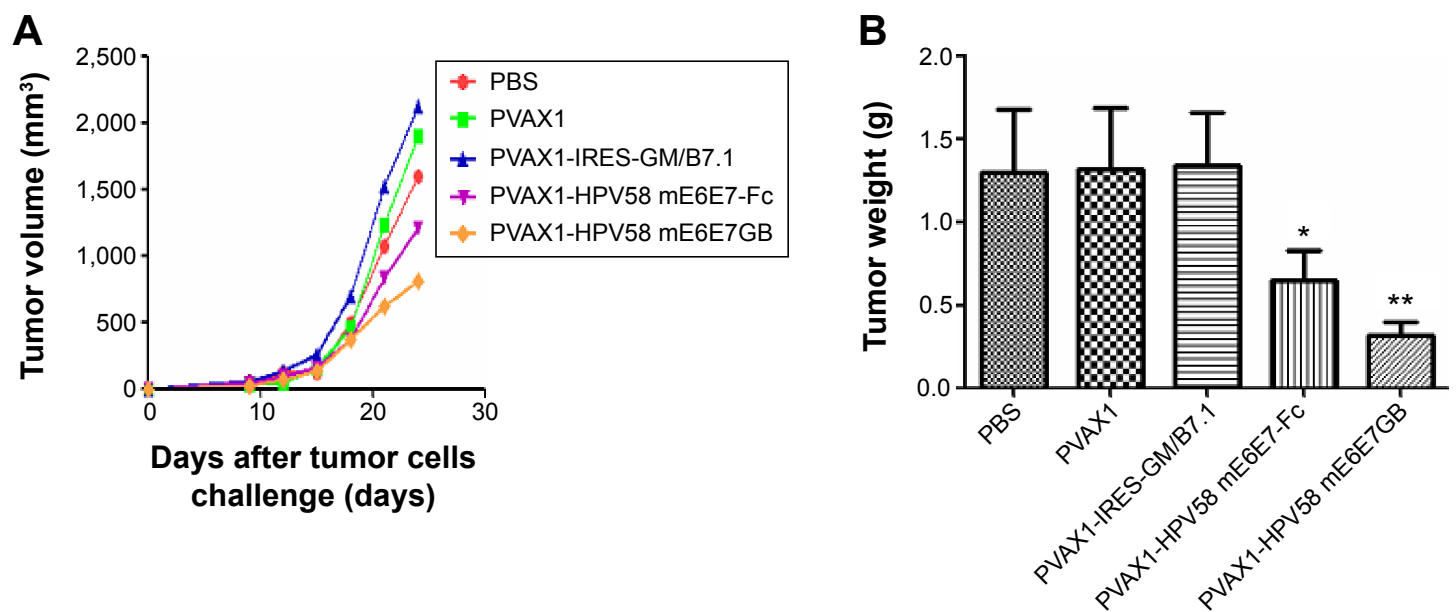

Figure 6 In vivo tumor growth inhibition experiments to compare the antitumor effects of various DNA plasmids against HPV58 E6E7-expressing BI6-HPV58 E6E7 tumors in vaccinated mice.

Notes: The graph shows the tumor growing curve $(\mathbf{A})$ and tumor mean weight $(\mathbf{B})$ on day 24 after BI6-HPV58 E6E7 cell challenge. (A) The tumor growing curve of the PVAXI-HPV58 mE6E7FcGB group was the lowest among the mice in the five groups. (B) The tumor weights from tumor-bearing mice after treatment are expressed as mean \pm SD. The mean tumors weight in the PVAXI-HPV58 mE6E7FcGB group $(0.26 \pm 0.1 \mathrm{lg})$ was lower than I.40 $\pm 0.52 \mathrm{~g}$ for the PBS group, I.50 $\pm 0.60 \mathrm{~g}$ for the PVAXI group, I.62 $\pm 0.3 \mathrm{I} \mathrm{g}$ for the PVAXI-IRES-GM/B7.I group, and $0.66 \pm 0.34 \mathrm{~g}$ for the PVAXI-HPV58 mE6E7Fc group, and these differences were significant $(P<0.0 \mathrm{I})$. $* P<0.05$, $* * p<0.01$.

Abbreviations: PBS, phosphate-buffered saline; SD, standard deviation; HPV, human papillomavirus. 


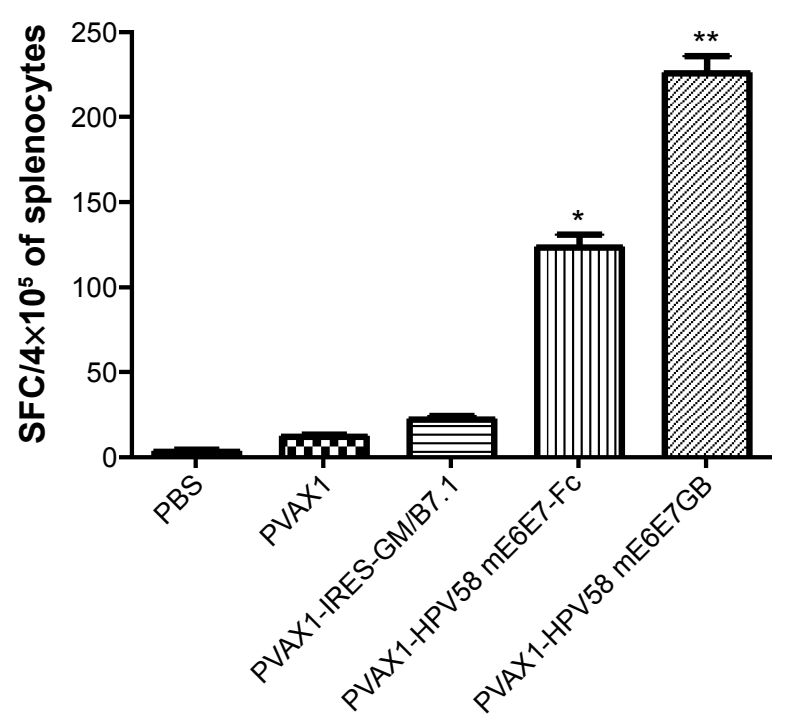

Figure 7 Specific T-cell responses were tested directly ex vivo in IFN- $\gamma$ Elispot assays against the HPV58 E6E7-GST protein.

Notes: The data shown are mean \pm standard deviation for three independent experiments. The average number of IFN- $\gamma$-positive spots was $218.75 \pm 34.42$ $\mathrm{SFC} / 4 \times 10^{5}$ splenocytes in the PVAXI-HPV58 mE6E7FcGB group. The positive spots were significantly enhanced compared with those of control mice immunized with PVAXI-HPV58 mE6E7Fc ( $138.6 I \pm 17.54 \mathrm{SFC} / 4 \times 10^{5}$ splenocytes, $\left.P<0.05\right)$, PVAXIIRES-GM/B7.I $\left(27.61 \pm 5.37 \mathrm{SFC} / 4 \times 10^{5}\right.$ splenocytes, $\left.P<0.01\right)$, PVAXI $(13.33 \pm 1.53$ SFC $/ 4 \times 10^{5}$ splenocytes, $\left.P<0.001\right)$, or PBS $\left(5.62 \pm 4.78 \mathrm{SFC} / 4 \times 10^{5}\right.$ splenocytes, $P<0.001)$. $* P<0.01$, ** $P<0.001$.

Abbreviations: IFN- $\gamma$, interferon; PBS, phosphate-buffered saline; SFC, spotforming cells; HPV, human papillomavirus.

\section{Discussion}

Persistent infection with high-risk HPV is a predominant cause of cervical cancer. Although HPV58 only accounts for $3.3 \%$ of cervical cancer cases globally ${ }^{25}$ it ranks as the third

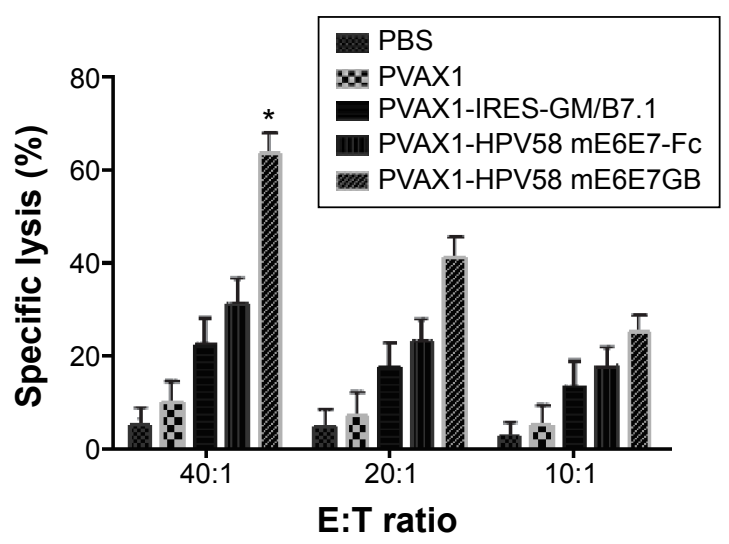

Figure $8 \mathrm{CTL}$ assays to analyze the lymphocytes from mice vaccinated with different DNA constructs using a quantitative LDH release assay.

Notes: Data were collected in the form of $L D H$ results at various E:T ratios (E:T $=40: I$, $20: 1,10: 1)$ and expressed as the percent cytotoxicity \pm SD. The lymphocytes from mice vaccinated with PVAXI-HPV58 mE6E7FcGB $(63.4 \% \pm 3.3 \%)$ had significantly higher specific cytolytic activity at an E:T ratio of $40: 1$ than lymphocytes from mice vaccinated with PVAXI-HPV58 mE6E7Fc (3I.1\% $\pm 2.75 \%)$, PVAXI-IRES-GM/B7.I (22.1\% $\pm 0.99 \%)$, PVAXI $(9.4 \% \pm 0.39 \%)$, or PBS $(5.3 \% \pm 2.2 \%)(P<0.05)$. The differences between all of these groups at E:T ratios of $20: 1$ or $10: 1$ did not reach overall statistical significance. ${ }^{*} P<0.05$.

Abbreviations: CTL, cytotoxic T lymphocyte; E:T, effector-to-target cell; HPV, human papillomavirus; LDH, lactate dehydrogenase; PBS, phosphate-buffered saline; $\mathrm{SD}$, standard deviation most common HPV type in Asia. ${ }^{11}$ Therefore, it is important to develop therapeutic vaccines for HPV58-associated neoplasms, such as cervical cancer and intraepithelial precursor lesions. Many preclinical studies suggest that a therapeutic immune-based vaccine strategy using E6 and/or E7 may be of value in the treatment of established disease. However, these studies primarily focused on HPV16 and $18,,^{23,26-28}$ and research on HPV58 therapeutic vaccines was rarely reported. ${ }^{29,30}$ In this study, we constructed a recombinant DNA vaccine, PVAX1HPV58 mE6E7FcGB, which can coexpress the HPV58 $\mathrm{E} 6 \mathrm{E} 7 \mathrm{Fc}$ fusion protein and the molecular adjuvants GM-CSF and B7.1 as a candidate therapeutic vaccine to treat HPV58.

All the HPV E6- and E7-encoding genetic vaccines used in clinical trials to date contain point mutations that prevent the binding of the encoded proteins to $\mathrm{p} 53$ and pRB, respectively. ${ }^{19,20,31}$ To ensure the safety of this vaccine for future clinical applications, we modified the six sites in the HPV58 E6 and E7 genes that are associated with their transforming properties (the p53- and pRB-binding sites) to inactivate their oncogenic activity, and we then fused these mutated genes into one reading frame. We found that HPV58 mE6E7 lacks the ability to transform human cells in vitro. Despite the lack of transformation activity, HPV58 $\mathrm{mE} 6 \mathrm{E} 7$ retains the ability to induce HPV-specific cell-mediated immune responses in vivo.

Several preclinical studies suggest that a therapeutic immune-based vaccine strategy using HPV58 E6 and/or E7 may be of value in the treatment of established disease. However, these published studies used vaccines based on specific regions of E6 and/or E7. ${ }^{32,33}$ By expressing slightly varied nononcogenic full-length forms of both HPV58 E6 and E7, our strategy has the advantage of maintaining native antigenic processing and thus theoretically increasing the opportunity for an immune response in vivo. Given the heterogeneity of immune responses in humans, which are much less predictable than those of inbred strains of mice, the expression of full-length proteins allows antigen processing that may produce a broad and effective antitumor response. Moreover, the foreign viral HPV58 mE6E7 antigens are "nonself" and have less potential to induce autoimmunity than other tumor-associated antigens.

Another promising approach to increase the potency of DNA vaccine is to link antigens to a human IgG Fc fragment. The Fc fragment in HPV58 mE6E7-Fc fusion proteins helps dendritic cells (DCs) to capture, process, and present the fusion protein via $\mathrm{Fc}$ receptor-mediated endocytosis. ${ }^{34,35}$ In addition, the Fc fragment not only enhances antibody-dependent cellmediated cytotoxicity and activation of phagocytic cells, but also prolongs the half-life of the HPV58 mE6E7-Fc fusion antigen. ${ }^{32}$ The glycosylphosphatidylinositol (GPI) moiety at the C-terminus anchors proteins to the eukaryotic cell membrane. 
The GPI molecule can be connected with a new protein in the endoplasmic reticulum cavity and form a GPI-anchored protein. When a GPI-anchored protein is incubated with cells, it can be automatically integrated into the cell membrane and maintain its biological activity. ${ }^{33}$ In this study, we linked the HPV58 mE6E7-Fc fusion antigen with a GPI-anchored protein to anchor the fusion antigen to the cell membrane to enhance the immune recognition of the antigen by specific lymphocytes.

Delivery of the sig-HPV58 mE6E7-Fc-GPI fusion antigen is also of critical importance to the success of the vaccine. We chose the PVAX1 DNA vector, which was designed for use in the development of DNA vaccines and allows highcopy-number replication in E. coli and high-level transient expression of the protein of interest in most mammalian cells. The PVAX1 vector is stable and has an extensively characterized safety profile. ${ }^{36,37}$

The new PVAX1-IRES-GM/B7.1 vector was successfully modified with the GM-CSF and B7.1 molecular adjuvants via IRES linking. ${ }^{38}$ The use of IRESs enables vectors to produce multiple products from a single transcriptional unit, thus eliminating the loss of gene expression caused by promoter competition or counterselection. ${ }^{39}$ Thus, when the sigHPV58 mE6E7-Fc-GPI fusion antigen sequence was inserted upstream of the IRES of the PVAX1-IRES-GM/B7.1 vector, the modified construct expressed the sig-HPV58 mE6E7-FcGPI, GM-CSF, and B7.1 proteins. GM-CSF has the ability to promote the maturation of precursor cells into DCs. Mature DCs generate tumor antigen epitopes for cross-presentation on human leukocyte antigen (HLA)-I molecules to stimulate $\mathrm{CD}^{+}$cytotoxic T-cells or for conventional presentation on HLA-II molecules to stimulate CD4 ${ }^{+}$helper T-cells. ${ }^{40-42}$ B7.1 (CD80) plays a critical costimulatory role in the activation of TCR-stimulated CTLs by binding to CD28. ${ }^{42}$

In this study, we demonstrated that the recombinant DNA vaccine PVAX1-HPV58 mE6E7FcGB successfully expressed the desired protein products in transfected cells. We also demonstrated that a specific IFN- $\gamma$-positive T-cell response to the HPV58 E6E7-GST protein was elicited in C57BL/6 mice immunized with the recombinant DNA vaccine in two in vitro assays. Furthermore, we also detected HPV58-specific lytic activity and antitumor responses in HPV58 (+) B16-HPV58 E6E7 tumor-challenged mice, in which tumor development was delayed. These data suggested that HPV58 mE6E7 was efficiently presented in C57BL/6 mice immunized with the PVAX1-HPV58 mE6E7FcGB DNA vaccine.

In conclusion, we modified the HPV58 E6 and E7 oncogenes to eliminate their transformation activity, thus minimizing their oncogenic potential and adapting them for DNA immunization. Based on the results of the modified E6 and E7 genes, we fused them with a signal peptide and with human IgG Fc and GPI fragments, and this sig-HPV58 mE6E7-Fc-GPI fusion antigen was found to have superior immunogenicity. Subsequently, we constructed a new DNA vaccine, PVAX1-HPV58 mE6E7FcGB, that coexpresses the sig-HPV58 mE6E7-Fc-GPI fusion antigen and the GM-CSF and B7.1 molecular adjuvants. We observed that the recombinant DNA vaccine was able to significantly restrain the growth of HPV58 (+) tumors and generate significant and functional cellular immunity in mice. These data provide a basis for further study of this recombinant DNA vaccine as a potential vaccine candidate.

It should be noted that although our data demonstrated that the mutations in E6 and E7 have eliminated their transformation activity, more safety data would be needed to ensure their lack of tumorogenesis, such as the cellular immortalization assay. ${ }^{28}$ In addition, it would be interesting to test whether this novel DNA vaccine could elicit anti-HPV58 neutralizing antibodies, which have been shown to play an important role in the prevention of HPV58-associatied cancer. ${ }^{43,44}$

\section{Acknowledgments}

We thank Dr Jinqi Yan, Liang Zhang, Yi Xiao, Qi Wang, and Fang Liu for expert advice on molecular biology, cell culture, and animal experiments. This work was supported by Guangxi Health Department Key Projects (200970), Guangxi Health Department Self-financing projects (Z2007177).

\section{Disclosure}

The authors report no conflicts of interest in this work.

\section{References}

1. Parkin DM, Bray F, Ferlay J, Pisani P. Global cancer statistics, 2002. CA Cancer J Clin. 2005;55:74-108.

2. Walboomers JM, Jacobs MV, Manos MM, et al. Human papillomavirus is a necessary cause of invasive cervical cancer worldwide. J Pathol. 1999;189:12-19.

3. Villa LL, Costa RL, Petta CA, et al. Prophylactic quadrivalent human papillomavirus (types 6, 11, 16, and 18) L1 virus-like particle vaccine in young women: a randomised double-blind placebo-controlled multicentre phase II efficacy trial. Lancet Oncol. 2005;6:271-278.

4. Harper DM, Franco EL, Wheeler C, et al; GlaxoSmithKline HPV Vaccine Study Group. Efficacy of a bivalent L1 virus-like particle vaccine in prevention of infection with human papillomavirus types 16 and 18 in young women: a randomised controlled trial. Lancet. 2004;364: 1757-1765.

5. Schiller JT, Castellsagué X, Villa LL, Hildesheim A. An update of prophylactic human papillomavirus L1 virus-like particle vaccine clinical trial results. Vaccine. 2008;26:53-61.

6. Clifford GM, Smith JS, Plummer M, Muñoz N, Franceschi S. Human papillomavirus types in invasive cervical cancer worldwide: a meta analysis. Br J Cancer. 2003;88:63-73.

7. Peng S, Trimble C, He L, et al. Characterization of HLA-A2-restricted HPV-16 E7-specific CD8(+) T-cell immune responses induced by DNA vaccines in HLA-A2 transgenic mice. Gene Ther. 2006;13:67-77.

8. Peng S, Ji H, Trimble C, et al. Development of a DNA vaccine targeting human papillomavirus type 16 oncoprotein E6. J Virol. 2004;78: 8468-8476. 
9. Ressing ME, Sette A, Brandt RM, et al. Human CTL epitopes encoded by human papillomavirus type $16 \mathrm{E} 6$ and E7 identified through in vivo and in vitro immunogenicity studies of HLA-A*0201-binding peptides. J Immunol. 1995;154:5934-5943.

10. Bao YP, Li N, Smith JS, Qiao YL; ACCPAB members. Human papillomavirus type distribution in women from Asia: a meta-analysis. Int J Gynecol Cancer. 2008;18:71-79.

11. Gao Q, Singh L, Kumar A, Srinivasan S, Wazer DE, Band V. Human papillomavirus type 16 E6-induced degradation of E6TP1 correlates with its ability to immortalize human mammary epithelial cells. $J$ Virol. 2001;75:4459-4466.

12. Munger K, Basile JR, Duensing S, et al. Biological activities and molecular targets of the human papillomavirus E7 oncoprotein. Oncogene. 2001;20:7888-7898.

13. Munger K, Baldwin A, Edwards KM, et al. Mechanisms of human papillomavirus-induced oncogenesis. J Virol. 2004;78:11451-11460.

14. Nguyen DX, Westbrook TF, McCance DJ. Human papillomavirus type 16 E7 maintains elevated levels of the cdc25A tyrosine phosphatase during deregulation of cell cycle arrest. J Virol. 2002;76:619-632. doi: 0.1128/JVI.76.2.619-632.2002.

15. Somasundaram C, Takarnatsu H, Andreoni C, et a1. Enhanced protective response and immuno-adjuvant effects of porcine GM-CSF on DNA vaccination of pigs against Aujeszky's disease virus. Vet Immunol Immunopathol. 1999;70(3-4):277-287.

16. Ou-Yang P, Hwang LH, Tac MH, et al. Co-delivery of GM-CSF gene enhances the immune responses of hepatitis $\mathrm{C}$ viral core protein-expressing DNA vaccine: role of dendritic cells. J Med Virol. 2002;66(3): 320-328.

17. Kim JJ, Nottingham LK, Wilson DM, et a1. Engineering DNA vaccines via co-delivery of co-stimulatory molecule genes. Vaccine. 1998;16(19): $1828-1835$

18. Zheng GX, Liu SR, Wang PJ, et al. Arming tumor-reactive T cells with costimulator B7.1 enhances therapeutic efficacy of the T cells. Cancer Res. 2006;66(13):6793-6799.

19. Zhou X, Qian X, Zhao Q, Lu Y, Xiong M. Efficient expression of modified human papillomavirus $16 \mathrm{E} 6 / \mathrm{E} 7$ fusion protein and the antitumor efficacy in a mouse model. Biol Pharm Bull. 2004;27:303-307.

20. Cassetti MC, McElhiney SP, Shahabi V, et al. Antitumor efficacy of Venezuelan equine encephalitis virus replicon particles encoding mutated HPV16 E6 and E7 genes. Vaccine. 2004;22:520-527.

21. Luo LQ, Li J, Zhang YH, et al. Transforming activity and immunogenicity of HPV58 E7. In: International Symposium on Biology of Cancer Prevention and Treatment, Beijing, 1999. Beijing: Chinese Society of Oncology, Chinese Medical Association.

22. McIntyre MC, Frattini MG, Grossman SR, Laimins LA. HPV 18 E7 protein requires intact Cys-X-X-Cys motifs for zinc binding, dimerization, and transformation but not for Rb binding. J Virol. 1993;67(6): 3142 .

23. Oosterhuis K, Ohlschläger $\mathrm{P}$, van den Berg JH, et al. Preclinical development of highly effective and safe DNA vaccines directed against HPV 16 E6 and E7. Int J Cancer. 2011;129:397-406.

24. Zhao KJ, Cheng H, Zhu KJ, et al. Recombined DNA vaccines encoding calreticulin linked to HPV6bE7 enhance immune response and inhibit angiogenic activity in B16 melanoma mouse model expressing HPV 6bE7 antigen. Arch Dermatol Res. 2006;298:64-72.

25. Smith JS, Lindsay L, Hoots B, et al. Human papillomavirus type distribution in invasive cervical cancer and high-grade cervical lesions: a meta-analysis update. Int J Cancer. 2007;121:621-632.

26. Karanam B, Gambhira R, Peng S, et al. Vaccination with HPV16L2E6E7 fusion protein in GPI-0100 adjuvant elicits protective humoral and cell-mediated immunity. Vaccine. 2009;27:1040-1049.
27. Zhao L, Liu B, Ren J, et al. Immunogenicity in mice and rhesus monkeys vaccinated with recombinant vaccinia virus expressing bivalent E7E6 fusion proteins from human papillomavirus types 16 and 18. Virol J.2011; 8:302-312.

28. Wieking BG, Vermeer DW, Spanos WC, et al. A non-oncogenic HPV 16 E6/E7 vaccine enhances treatment of HPV expressing tumors. Cancer Gene Ther. 2012;19:667-674.

29. Luo LQ, Li J, Zhang YH, Zhang YH. Experimental study of the immuno-protective activity of recombinant vaccinia virus expressing HPV58 E7. Zhongguo Yi Xue Ke Xue Yuan Xue Bao. 2003;25: $43-46$.

30. Deng D, Liao S, Bai X, et al. The preparation of human papillomavirus type 58 vaccine and exploring its biological activity and immunogenicity in vitro. Int J Gynecol Cancer. 2011;21:988-995.

31. Hung CF, Monie A, Alvarez RD, Wu TC. DNA vaccines for cervical cancer: from bench to bedside. Exp Mol Med. 2007;39:679-689.

32. Ferrone CR, Perales MA, Goldberg SM, et al. Adjuvanticity of plasmid DNA encoding cytokines fused to immunoglobulin Fc domains. Clin Cancer Res. 2006;12:5511-5519.

33. Ikezawa H. Glycosylphosphatidylinositol (GPI)-anchored proteins. Biol Pharm Bull. 2002;25:409-417.

34. Rafiq K, Bergtold A, Clynes R. Immune complex-mediated antigen presentation induces tumor immunity. J Clin Invest. 2002;110:71-79.

35. Liu R, Zhou C, Wang D, et al. Enhancement of DNA vaccine potency by sandwiching antigen-coding gene between secondary lymphoid tissue chemokine (SLC) and IgG Fc fragment genes. Cancer Biol Ther. 2006; $5: 427-434$

36. Ma $\mathrm{CH}$, Zhang $\mathrm{Y}$, Wang $\mathrm{XY}$, et al. Human endostain gene transfer. Either naked or with liposome, has the same inhibitory effect on growth of mouse liver tumor cells in vivo. World J Gastroenterol. 2004;10(19): 2874-2877.

37. Li JW, Gong JY, Li K, et al. Construction of a fusion expression plasmid containing the G250 gene and human granulocyte-macrophage colony stimulating factor and its significance in renal cell carcinoma. Oncol Lett. 2011;2(2):343-347.

38. Liu N, Yan J, Ran DL, et al. Cloning and expression of fusion gene of human GM-CSF and B7.1 in bicistronic eukaryotic vector pVAX1IRES. Bull Acad Mil Med Sci. 2008:32:249-252.

39. Allera-Moreau C, Chomarat P, Audinot V, et al. The use of IRESbased bicistronic vectors allows the stable expression of recombinant G-protein coupled receptors such as NPY5 and histamine 4. Biochimie. 2006;88:737-746.

40. Pan PY, Li Y, Li Q, et al. In situ recruitment of antigen-presenting cells by intratumoral GM-CSF gene delivery. Cancer Immunol Immunother. 2004;53:17-25.

41. Mellman I, Steinman RM. Dendritic cells: specialized and regulated antigen processing machines. Cell. 2001;106:255-258.

42. Ren SP, Wu CT, Huang WR, et al. Adenoviral-mediated transfer of human wild-type p53, GM-CSF and B7.1 genes results in growth suppression and autologous anti-tumor cytotoxicity of multiple myeloma cells in vitro. Cancer Immunol Immunother. 2006;55: 375-385.

43. Scherpenisse M, Schepp RM, Mollers M, Meijer CJ, Berbers GA, van der Klis FR. Characteristics of HPV-specific antibody responses induced by infection and vaccination: cross-reactivity, neutralizing activity, avidity and IgG subclasses. PLoS One. 2013;8(9):797.

44. Draper E, Bissett SL, Howell-Jones R, et al. A randomized, observerblinded immunogenicity trial of Cervarix and Gardasil human papillomavirus vaccines in 12-15 year old girls. PLoS One. 2013;8(9): 861-825. 
OncoTargets and Therapy

\section{Publish your work in this journal}

OncoTargets and Therapy is an international, peer-reviewed, open access journal focusing on the pathological basis of all cancers, potential targets for therapy and treatment protocols employed to improve the management of cancer patients. The journal also focuses on the impact of management programs and new therapeutic agents and protocols on

perspectives such as quality of life, adherence and satisfaction. The manuscript management system is completely online and includes a very quick and fair peer-review system, which is all easy to use. Visit http://www.dovepress.com/testimonials.php to read real quotes from published authors.

Submit your manuscript here: http://www.dovepress.com/oncotargets-and-therapy-journal 